\title{
PERSPECTIVE \\ Utilizing Lean Leadership Principles to Build an Academic Primary Care Practice of the Future
}

\author{
Sasha Morduchowicz, MPH' , Jonathan S. Lee, MD, MAS² (D), Lei Choi, MD, MPH², \\ Coleen Kivlahan, $\mathrm{MD}^{3}$, Dan Null, $\mathrm{MD}^{2}$, Susan Smith, $\mathrm{MD}^{7}$, and Mitchell D. Feldman, \\ MD, MPhil
}

${ }^{1}$ Faculty Practice Operations \& Ambulatory Services, University of California, San Francisco, San Francisco, CA, USA; ${ }^{2}$ Division of General Internal Medicine, University of California, San Francisco, San Francisco, CA, USA; ${ }^{3}$ Primary Care Services, University of California, San Francisco, San Francisco, CA, USA.

This Perspective presents a case study of multidimensional clinical transformation in an academic general internal medicine practice. In the face of increasing internal and external pressures, health systems and individual medical practices have pursued multiple strategies to improve quality, patient experience, and efficiency, while reducing staff and provider stress and burnout. We describe a Lean-informed approach that emphasizes the importance of organizational alignment in goals, evidence-based problem solving, and leadership behaviors to support a culture of continuous improvement. Our aim in this Perspective is to provide a real-world example of a feasible process for the planning, preparation, and execution of effective transformation, and to present lessons that may be useful to other academic health center practices seeking to develop innovative models to achieve the quadruple aim.

KEY WORDS: academic transformation; Lean; continuous improvement; leadership behaviors; change management; burnout.

J Gen Intern Med 35(12):3650-5

DOI: $10.1007 / \mathrm{s} 11606-020-06246-7$

(C) Society of General Internal Medicine 2020

\section{INTRODUCTION}

Academic health center practices face increasing pressures to improve the quality of care and patient and provider experience while reducing health care costs. ${ }^{1}$ These pressures are exacerbated by the high prevalence and negative impacts of provider burnout in academic primary care. ${ }^{2,3}$ Addressing these challenges requires innovative approaches aimed at the transformation of primary care.

In this Perspective, we describe a case study in one academic primary care practice that has launched a multi-year, Lean-informed, transformation. We describe our impetus for change, key guiding principles, and leadership engagement and change management strategies. Early learnings that may

Sasha Morduchowicz and Jonathan S. Lee are co-first authors.

Received August 28, 2019

Accepted September 14, 2020

Published online September 28, 2020 inform other academic general internal medicine practices considering similar transformation efforts are also discussed.

\section{Impetus for Change}

Amid concerns about faculty burnout, leaders at the Division of General Internal Medicine (DGIM) at the University of California, San Francisco (UCSF), surveyed physicianfaculty in 2015 to assess symptoms of burnout and work conditions using the Mini-Z Burnout Survey., ${ }^{2}$ When compared with national survey results from other academic general internal medicine divisions, self-reported burnout rates at DGIM were among the highest. ${ }^{4} \mathrm{~A}$ taskforce was convened and found that burnout was a symptom of deeper root causes within the clinic, including a chaotic work environment, siloed workflows, poor team communication, and duplication and delays in responding to clinical messages, leading to excessive documentation burden.

Concurrently, UCSF was beginning to implement the Lean management system across the health system and leadership wanted a site to pilot practice transformation efforts with the goal of learning from this experience to spread similar transformation efforts across the organization. Based on DGIM's preliminary results and the taskforce's findings, health system and DGIM leadership decided to launch a multi-year transformation using Lean as an improvement methodology and management system.

\section{GUIDING PRINCIPLES}

For our Lean-informed approach, we applied an existing definition of Lean as an "overall management/operating system that uses a continuous improvement culture that empowers frontline workers (nurses, physicians, other caregivers and staff) to solve problems and eliminate waste by standardizing work to improve the value of care delivered to patients." ${ }^{.5}$ This definition recognizes Lean as a management system that encompasses the social, behavioral, and contextual organizational factors that enable change and empower teams rather than just a technical methodology focused on process improvement tools. ${ }^{6-8}$ Health system leadership developed 
guiding principles centered on organizational alignment, adoption of key leadership behaviors, and evidence-based problem solving that correspond to the broader Lean principles of align, enable, and improve. ${ }^{9}$

While we opted to use Lean to guide our efforts, it is important to recognize that other quality improvement and transformation approaches share similar guiding principles. ${ }^{10}$,

${ }^{11}$ Our view is that the specific methodology is less important than choosing an approach that moves beyond the technical and emphasizes the social and behavioral components of quality improvement. The goal is to develop a culture where improvement work is championed by frontline workers and recognized as part of everyday work rather than just part of discrete improvement projects. When applied in this way, Lean and other methodologies have the potential to sustain effective change beyond the initial transformation period. ${ }^{12,13}$

To build this culture of problem solving and continuous improvement, the Lean system requires leaders to embody and adopt key behaviors. ${ }^{10}$ For example, leaders were trained to ask, "What problem are we trying to solve?" rather than jumping to a proposed solution without first ensuring that the problem was understood. Similarly, to create the necessary conditions for developing the problem solving capabilities of frontline providers and staff, leaders were coached to ask open-ended questions to elicit ideas from the frontline personnel rather than ask leading questions with embedded solutions. Over the course of the practice transformation work at DGIM, leaders were trained and coached to practice these directly observable behaviors. The importance of developing this culture of improvement cannot be understated. Ultimately, this is likely the most important and correspondingly most difficult change to affect in the Lean management system. Leaders must understand early on that the consistency and quality of their behavior are vital in this process to build the culture and sustain change.

DGIM leaders outlined additional principles corresponding to the academic medical center mission including integrating the learning health system into the transformation process and reducing health disparities. For example, as part of the focus on a learning health system, leaders intentionally planned around how residents and trainees would be involved and what impact that would have from an educational standpoint. Residency program leaders also participated in each leadership visioning event and residents participated in the improvement events described below. Similarly, as part of the focus on reducing health disparities, attention was paid at each step to how changes in workflows would impact underserved clinic patients.

\section{LEADERSHIP SITE VISITS}

To lay the foundation for change, leaders conducted a series of site visits to other health care organizations. The goals were (1) to witness how lean principles and management systems operate in action and (2) to identify specific elements to implement at UCSF. A group of over twenty UCSF leaders, including C-suite representatives, visited three health care systems undergoing similar primary care transformation efforts. UCSF conducted pre-meetings with each organization to plan the agendas which included small group breakouts to explore different clinic locations and topics. Each small group was assigned a UCSF facilitator tasked with taking notes to bring back to the larger group. After the visits, UCSF leaders held debrief meetings to discuss the care delivery models, improvement methodologies, and management structures they had observed to decide which elements they wanted to bring back to UCSF.

\section{VALUE STREAM MAPPING EVENT: UNDERSTANDING THE CURRENT STATE}

Clear articulation of the problems to be solved and a deep understanding of the current state (i.e., what is happening today) are critical first steps in any Lean improvement work. Consequently, the DGIM team first conducted a 5-day value stream mapping (VSM) event. VSM is a Lean tool used to evaluate and visually depict an entire process through direct observation and distinguishes whether discrete process steps do or do not contribute value from the customer's (i.e., patient's) perspective. ${ }^{11}$ Within an academic medical center, the patient may not always be the "customer" (i.e., the target or beneficiary of the intervention). In assessing the learning environment, the trainees are sometimes the customers and the assessment of value is viewed from their perspective.

During the DGIM VSM workshop, detailed visual maps were created for (1) providing in-person care to patients, (2) scheduling patient appointments, (3) processing refills, (4) reviewing and communicating results, and (5) receiving and responding to messages regarding advice and symptoms (Fig. 1). Thirty participants conducted the VSM including primary care leaders, physician leaders, frontline providers, clinical and administrative staff, and internal medicine residents. A UCSF Lean consultant team provided education and facilitation support. An Epic team provided real-time electronic health record (EHR) data reporting and analytic support.

Importantly, senior leaders were directly involved in the collection of first-hand, observable evidence during process observations. Seeing the work where it takes place, in this case the clinic and the EHR, is an activity referred to in Lean as a "gemba walk." Gemba is a Japanese word meaning where the work takes place. This level of involvement from senior leaders was critically important because it enabled them to role model leadership behaviors including leading with humility and continuous learning.

The VSM team found that in-clinic visit and virtual EHR inbasket work took place in silos and that there was variability in how the work was approached by both providers and staff. This ultimately led to delays and inconsistencies in the 


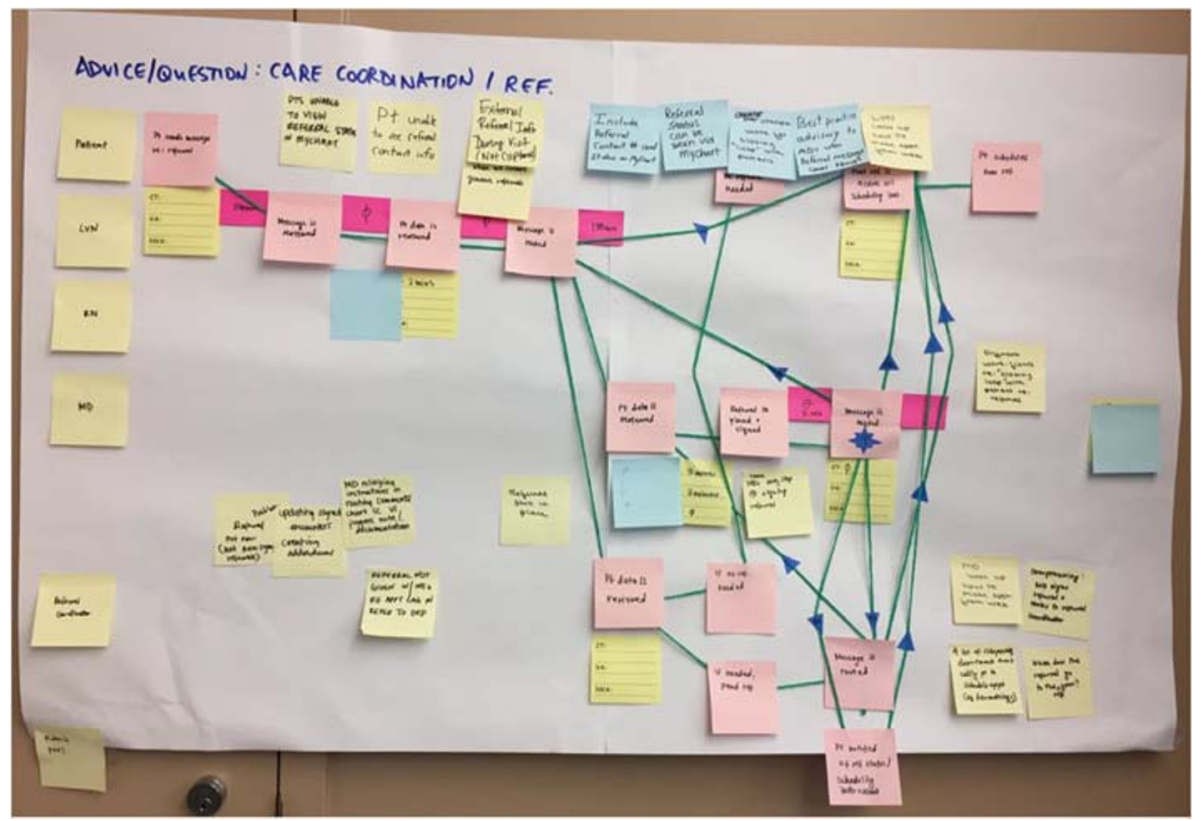

Figure 1 Sample DGIM value stream map. This value stream map depicts the process of receiving and responding to electronic patient messages and was created by a multidisciplinary team including primary care and physician leaders, frontline providers, clinical and administrative staff, internal medicine residents, and Lean and electronic health record consultants.

experience of care for patients. One key finding was that it took on average seven touches by various members of the team and six calendar days to resolve in-basket patient advice messages. These delays often generated duplicate patient messages and unnecessary work and were a major contributor to patient dissatisfaction. A unique challenge and contributor to the findings was variation in clinical FTEs of providers with different roles (primarily clinician vs researcher vs educator) within an academic medical center. For some metrics, we found it helpful to stratify the data by different provider phenotypes based on their clinical FTE.

\section{A DATA-DRIVEN JOURNEY: ADDITIONAL CURRENT STATE ASSESSMENT ACTIVITIES}

Following the VSM, DGIM leaders further identified a comprehensive set of data metrics needed to deepen their understanding of the current state. Quantitative data was collected by a team of leaders, internal and external Lean consultants, and a data analyst, all funded by health system leadership (Table 1). Qualitative data was also collected in the form of semi-structured interviews with patients, providers, and clinical staff about the in-clinic experience and virtual work (Table 2). The cumulative data story that emerged represented a comprehensive baseline view of the current state and highlighted the most critical areas for improvement.

Similar to the VSM, the DGIM Chief and Medical Director participated in the observations and interviews. This was an intentional part of the change management strategy. Going to see the work and interviewing the people who do the work was one way of showing respect for every individual.

\section{LEADERSHIP VISIONING EVENT}

In August of 2017, a group of senior leaders convened to review the extensive findings from the VSM and current state

Table 1 Current State Analysis Questions and Metrics

\begin{tabular}{|c|c|c|}
\hline$\overline{\text { Topic }}$ & Sample metrics & Data sources \\
\hline $\begin{array}{l}\text { Clinical and } \\
\text { administrative } \\
\text { team information }\end{array}$ & $\begin{array}{l}\text { - Number of faculty, } \\
\text { residents, MAs, LVNs, } \\
\text { RNs, front office staff, } \\
\text { and schedulers }\end{array}$ & $\begin{array}{l}\text { - Health system, } \\
\text { division and } \\
\text { residency rosters }\end{array}$ \\
\hline $\begin{array}{l}\text { Patient } \\
\text { demographics }\end{array}$ & $\begin{array}{l}\text { - Number of empaneled } \\
\text { patients, age, payor mix, } \\
\text { gender, race, ethnicity } \\
\text { and language, complexity } \\
\text { and distribution across } \\
\text { providers, panel size }\end{array}$ & $\begin{array}{l}\text { - Electronic health } \\
\text { record reports }\end{array}$ \\
\hline Access & $\begin{array}{l}\text { - Patient and provider } \\
\text { continuity } \\
\text { - Third Next available } \\
\text { appointment (3NA) } \\
\text { - Backlog: number of } \\
\text { patient visits booked into } \\
\text { the future per provider } \\
\text { - Schedule Leveling: } \\
\text { number of providers in } \\
\text { clinic per day (including } \\
\text { staffing and room ratios) }\end{array}$ & $\begin{array}{l}\text { - Electronic health } \\
\text { record reports }\end{array}$ \\
\hline In-clinic & $\begin{array}{l}\text { - Common interruptions } \\
\text { or "flow busters" }\end{array}$ & $\begin{array}{l}\text { - Hand tallied by } \\
\text { providers, clinical } \\
\text { and administrative } \\
\text { staff }\end{array}$ \\
\hline $\begin{array}{l}\text { Call } \\
\text { management }\end{array}$ & - Reasons for patient calls & $\begin{array}{l}\text { - Call center reports } \\
\text { - Direct } \\
\text { observations }\end{array}$ \\
\hline In-basket & $\begin{array}{l}\text { - Daily volumes by type } \\
\text { of message } \\
\text { - Number of touches to } \\
\text { resolve a message by type } \\
\text { - Turnaround times (in } \\
\text { hours) to resolve } \\
\text { messages by type }\end{array}$ & $\begin{array}{l}\text { - Electronic health } \\
\text { record reports } \\
\text { - Direct } \\
\text { observations }\end{array}$ \\
\hline
\end{tabular}


Table 2 Current State Analysis: Semi-structured Interview Questions

\begin{tabular}{|c|c|}
\hline Audience & Sample of key questions \\
\hline $\begin{array}{l}\text { Faculty (including preceptors) and nurse } \\
\text { practitioners }\end{array}$ & $\begin{array}{l}\text { - When it is a great day, what makes it a great day? } \\
\text { - What are your pain points in clinic? What about the in-basket is painful to you? What about the refill } \\
\text { process is painful to you? } \\
\text { - What is your wish for this improvement work? } \\
\text { - What concerns do you have about this improvement work? } \\
\text { - How would you describe your partnership with the back office staff? How does information flow? }\end{array}$ \\
\hline Front office staff & $\begin{array}{l}\text { - When it is a great day, what makes it a great day? } \\
\text { process is painful to you? } \\
\text { - What is your wish for this improvement work? } \\
\text { - What concerns do you have about this improvement work? }\end{array}$ \\
\hline Back office staff & $\begin{array}{l}\text { - When it is a great day, what makes it a great day? } \\
\text { process is painful to you? What is it like when providers run behind? } \\
\text { - What is your wish for this improvement work? } \\
\text { - What concerns do you have about this improvement work? }\end{array}$ \\
\hline Patients & $\begin{array}{l}\text { - What makes a great visit with your care team? } \\
\text { - What could be improved? } \\
\text { - How was your appointing experience? When did you make your appointment? } \\
\text { Patients paneled with residents: } \\
\text { - What attracts you to choose a resident as your PCP? } \\
\text { - Tell me about what it has been like communicating with your provider and team in between visits. } \\
\text { - What do you want when you transfer resident PCPs? What gives you confidence in the handoff of care? }\end{array}$ \\
\hline
\end{tabular}

assessment. The agenda (Table 3) included time to reflect on the key lessons learned from the VSM event and the site visits and to confirm a concrete vision for developing the practice of the future. The key deliverables for the event included a future state map outlining the areas of improvement to address over the multi-year initiative, a set of key performance metrics, a high-level implementation plan (including resource commitments), and a communication plan. Senior leadership committed resources and also placed limits on the scope of the work, based on available time and funding.

This foundational visioning event enabled the team to emerge with a clear set of priorities that were aligned with the broader organization's strategic vision, an important factor for future success. ${ }^{14}$ The group determined that the five most important areas to address as part of the transformation efforts were (1) patient telephone call management, (2) patient visit access, (3) leader standard work (LSW), (4) in-clinic patient flow, and (5) efficient management of EHR in-basket work. Each topic would be the focus of a week-long kaizen (i.e., rapidimprovement event); consequently, five kaizens were scheduled
Table 3 Leadership Visioning Event Agenda

\begin{tabular}{cl}
\hline \hline Day 1 & Agenda \\
8:00 & Welcome \& kick-off to set the context for change \\
8:30 & Value stream mapping event summary \\
9:00 & Share data on the current state \\
9:30 & Site visit reflections \& lessons learned \\
9:45 & Lean education-What is Lean? \\
10:30 & Break \\
10:45 & Conceptual visioning \\
11:30 & Conceptual visioning group report out \& discussion \\
12:00 & Lunch \\
12:30 & Assumptions for future state design \& Lean value stream \\
& mapping and context \\
12:45 & Future state mapping: managing supply and demand \& clinic \\
& flow \\
5:00 & Adjourn \\
Day 2 & Agenda \\
8:00 & Kickoff \& reflections from day 1 \\
8:40 & Future state mapping continued \\
11:00 & Review future state map \\
12:00 & Lunch \\
12:45 & Improvement grouping \& sequencing the work \\
1:45 & Breakout groups: develop key metrics for each body of work \\
$2: 45$ & Break \\
$3: 00$ & Small group report outs \\
$3: 30$ & Communication planning \\
$4: 30$ & Wrap up \\
5:00 & Adjourn \\
\hline &
\end{tabular}


from 2017 to 2019 (Fig. 2). The vision of the overall initiative was to re-imagine the entire model of care for in-clinic and virtual work, and to develop the management system to implement, sustain, and continuously improve the work. By the end of the leadership visioning session, an overarching plan had been developed, outlining commitment of time and resources to support the multi-year improvement effort.

\section{DISCUSSION AND LESSONS LEARNED}

Several key insights emerged from the initial work that we hope will inform other academic health centers considering transformation efforts.

\section{Lesson 1: Alignment at all Organizational Levels Is Key}

Alignment at all levels of the organization is needed to engage in a comprehensive transformation effort that moves beyond incremental, piecemeal improvement. Leaders at every level of the organization, from the Chief Executive Officer to the frontline staff, have to embrace change in order to make impactful and sustainable improvements. ${ }^{15}$ In addition, without the commitment, involvement, and sponsorship of executive leaders from the start, major clinical transformation is unlikely to be successful. ${ }^{5}$

One critical component of organizational alignment is the identification and establishment of well-articulated goals representing the vital few priorities that are most important to the organization. In Lean, these goals are referred to as True North goals. UCSF Health set True North goals in 2016 with key performance metrics focused on six domains: patient experience, quality and safety, provider and staff satisfaction, financial strength, strategic growth, and a learning health system. This alignment and constancy of purpose was critical to establish before starting the DGIM work because it enabled the design of the future state vision to align with UCSF's True North goals.

\section{Lesson 2: A Rigorous, Data-Driven Approach Will Support the Business Case}

The depth of the quantitative and qualitative evidence supporting the need for change was likely a major factor in executive leaders' willingness to commit time and resources to this large-scale transformation effort. Much of this evidence was gathered during the VSM event, highlighting the importance of a well-organized and conducted VSM as a key early step in the transformation process. For example, the awareness that it took DGIM on average seven touches by various members of the team and six calendar days to resolve patient telephone messages led the sponsors to prioritize call management.

As part of the VSM, senior leaders also directly observed clinical work on the frontlines (i.e., a gemba walk) to better understand the day-to-day work and challenges faced by staff and providers. We believe these experiences were critical and further contributed to leadership commitment to the improvement work. Reserving time for physician leaders, particularly in an academic setting, to be present for meetings and observations can be challenging. Purposeful and focused observations in key clinical areas are crucial. Qualitative interviews with leadership following the VSM event may have been particularly informative and should be considered as part of the transformation process.

\section{Lesson 3: Effective Change Management Requires an Intentional Strategy and Communication Plan}

Integrating new processes and behaviors into a complex academic delivery system is challenging and requires preparation, communication, and an intentional and dynamic change management approach. Senior leaders, coached by the internal and external quality improvement consultant team, were tasked with communicating updates and important messages to providers and staff. Leaders communicated in-person at group meetings, by e-mail, and by way of a weekly newsletter created specifically for the initial transformation work. One key message that needed to be communicated early on was to dispel the notion that this would be a discrete improvement project with clear starting and stopping points. Instead, the goal was a cultural transformation where improvement work would become a continuous part of daily work.

To prepare providers and staff for change, we needed to motivate them to take on the challenge of practice transformation. Whereas quantitative data was essential in obtaining commitment from health system leadership, we found that,

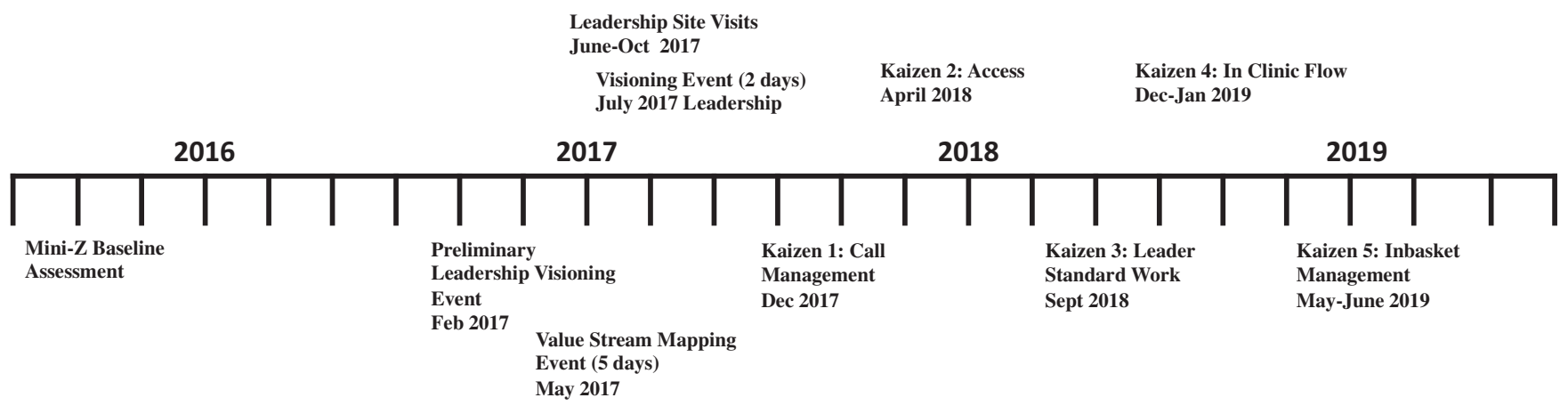

Figure 2 Transformation event timeline. 
for frontline providers and staff, focusing on the direct emotional connection to the work and its impact on coworkers and patients was a more effective motivator than quantitative data alone.

Despite our intentional communication strategies, we still encountered challenges. In particular, though leaders intentionally integrated frontline recommendations into the visioning process, some staff and providers felt as if the new model of care had already been designed by leaders before the initiative had even launched. It was important to clarify that the role of senior leaders was to describe the purpose (the why) and to determine the high-level future state vision (the what). While some aspects of the future state model of care were determined by the leaders (e.g., the staffing models impacting several departments beyond DGIM), the majority of the new work processes (the how) were to be designed by the frontline providers and staff during the kaizens. Direct participation in designing the work is a key component of increasing engagement among frontline workers and sustaining change. ${ }^{12} \mathrm{We}$ could have better addressed this concern by transparently communicating the role of each level of leadership at the outset of the effort.

\section{NEXT STEPS AND SUMMARY}

All five kaizen events have been completed and will be described in future manuscripts as outcomes are assessed. In this Perspective, our aim was to describe our approach and early lessons learned, particularly those focused on leadership, that may be useful to other academic primary care practices considering similar transformation efforts. We also describe some challenges to implementation unique to academic health centers.

In summary, while it is not essential to adopt Lean in order to take on the kind of transformation work launched at DGIM, we believe that whatever methodology is employed must align with health system priorities, identify and use a rigorous, datadriven improvement methodology, and be intentional about communicating change to frontline workers. It is also important to move beyond the tools and technical aspects of improvement, and to embrace a larger set of principles and leadership behaviors that develop a shared culture that encourages problem solving. Sustainable change will likely depend on leadership commitment to these behaviors and the successful establishment of the shared culture.

Corresponding Author: Jonathan S. Lee, MD, MAS; Division of General Internal Medicine, University of California, San Francisco, ?San Francisco, CA, USA (e-mail: jonathan.lee2@ucsf.edu).

Author Contributions Sasha Morduchowicz had full access to all of the data in the study and takes responsibility for the integrity of the data and the accuracy of the data analysis.
Study concept and design: Sasha Morduchowicz, MPH; Lei Choi, MD, MPH; Jonathan S. Lee, MD, MAS; Coleen Kivlahan, MD; Dan Null, MD; Susan Smith, MD; Mitchell D. Feldman, MD, MPhil.

Drafting of the manuscript: Sasha Morduchowicz, MPH; Jonathan S. Lee, MD, MAS; Lei Choi, MD.

Critical revision of the manuscript for important intellectual content: Sasha Morduchowicz, MPH; Lei Choi, MD, MPH; Jonathan S. Lee, MD, MAS; Coleen Kivlahan, MD; Dan Null, MD; Susan Smith, MD; Mitchell D. Feldman, MD, MPhil.

Funding This study is funded by the Faculty Practice Office \& Ambulatory Services, UCSF Health and the Continuous Improvement Department, UCSF Health.

\section{Compliance with Ethical Standards:}

Conflict of Interest: The authors declare that they do not have a conflict of interest.

\section{REFERENCES}

1. Bodenheimer T, Sinsky C. From Triple to Quadruple Aim: Care of the Patient Requires Care of the Provider. Ann Fam Med 2014;12(6):573-576.

2. Linzer M, Poplau S, Babbott S, et al. Worklife and Wellness in Academic General Internal Medicine: Results from a National Survey. J Gen Intern Med 2016;31(9):1004-1010.

3. American Medical Association Steps Forward. Physician Burnout. https://edhub.ama-assn.org/steps-forward. Accessed 21 Sept 2020.

4. Lee JS, Karliner LS, Julian KA, Linzer M, Feldman MD. Change in Faculty Perceptions of Burnout and Work Life in an Academic General Medicine Clinic: A Pre-Post Study. J Gen Intern Med 2019;34(10):19731974.

5. Shortell SM, Blodgett JC, Rundall TG, Kralovec P. Use of Lean and Related Transformational Performance Improvement Systems in Hospitals in the United States: Results from a National Survey. Jt Comm J Qual Patient Saf 2018;44(10):574-582.

6. Al-Balushi S, Sohal AS, Singh PJ, Al Hajri A, Al Farsi YM, Al Abri R Readiness Factors for Lean Implementation in Healthcare Settings-a Literature Review. J Health Organ Manag 2014;28(2):135-153.

7. Grove AL, Meredith JO, Macintyre M, Angelis $\mathbf{J}$, Neailey $\mathbf{K}$. Lean Implementation in Primary Care Health Visiting Services in National Health Service Uk. Qual Saf Health Care 2010;19(5):e43.

8. Hung D, Gray C, Martinez M, Schmittdiel J, Harrison MI. Acceptance of Lean Redesigns in Primary Care: A Contextual Analysis. Health Care Manag Rev 2017;42(3):203-212.

9. Utah State University Shingo Institute. Shingo Model Handbook. 2014. http://sapartners.com/wp-content/uploads/2014/04/ShingoModelHandbook.pdf. Accessed June 18, 2020.

10. Institute for Healthcare Improvement. The Breakthrough Series: IHI's Collaborative Model for Achieving Breakthrough Improvement. IHI Innovation Series White Paper. 2003. www.IHI.org. Accessed 21 Sept 2020.

11. Scoville R, Little K. Comparing Lean and Quality Improvement. IHI White Paper. 2014. www.IHI.org. Accessed 21 Sept 2020.

12. Hung DY, Gray CP, Truong QA, Harrison MI. Sustainment of Lean Redesigns for Primary Care Teams. Qual Manag Health Care 2019;28(1): 15-24.

13. Hung DY, Harrison MI, Truong $\mathbf{Q}, \mathbf{D u} \mathbf{X}$. Experiences of Primary Care Physicians and Staff Following Lean Workflow Redesign. BMC Health Serv Res 2018;18(1):274.

14. Clack L, Zingg W, Saint S, et al. Implementing Infection Prevention Practices across European Hospitals: An in-Depth Qualitative Assessment. BMJ Qual Saf 2018;27(10):771-780.

15. Wagner EH, Gupta R, Coleman K. Practice Transformation in the Safety Net Medical Home Initiative: A Qualitative Look. Med Care 2014;52(11 Suppl 4):S18-22.

Publisher's Note: Springer Nature remains neutral with regard to jurisdictional claims in published maps and institutional affiliations. 